\title{
Damage Identification of a Steel Frame Based on Integration of Time Series and Neural Network under Varying Temperatures
}

\author{
Minshui Huang $\left(\mathbb{D}\right.$, Wei Zhao, Jianfeng Gu ${ }^{D}$, and Yongzhi Lei \\ School of Civil Engineering and Architecture, Wuhan Institute of Technology, Wuhan 430073, China \\ Correspondence should be addressed to Jianfeng Gu; gujianfeng.12345@163.com
}

Received 18 October 2019; Accepted 31 July 2020; Published 17 August 2020

Academic Editor: Dongsheng Li

Copyright (c) 2020 Minshui Huang et al. This is an open access article distributed under the Creative Commons Attribution License, which permits unrestricted use, distribution, and reproduction in any medium, provided the original work is properly cited.

\begin{abstract}
The effect of varying temperatures is one of the most important challenges of vibration-based damage identification due to its bigger effects on the structural response than the damage itself. This study presents a methodology incorporating the autoregressive (AR) time series model with two-step artificial neural networks (ANNs) to identify damage under temperature variations. AR coefficients, which are extracted by fitting the AR models to acceleration responses, are however sensitive to temperature changes, resulting in false diagnoses. Thus, two-step ANN models with the inputs of difference in AR coefficients are utilized to compensate the detrimental temperature variations. Finite element (FE) models of a steel-braced frame structure, simulating several damage scenarios with different damage locations and severities at fluctuating temperatures, are used to verify the effectiveness and reliability of this approach. Numerical results indicate that the proposed approach could successfully recognize, locate, and quantify damage by using output-only vibration and temperature data regardless of varying temperatures and noise perturbations.
\end{abstract}

\section{Introduction}

Structural health monitoring (SHM) has become a very important research area for evaluating the performance of critical civil infrastructure systems [1]. Damage identification is regarded as a crucial component of SHM; enormous techniques including localized methods and global methods have been developed and applied to large complex structures. Among them, vibration-based methods, which track the dynamic features of bridges to monitor the condition and assess damage of structures by using sensors, have been widely utilized for a few decades [2]. The changes of modal parameters or other damage-sensitive features based on the dynamic characteristics of civil structures can be used to indicate the existence, location, and severity of damage. However, the fact that environmental effects (such as temperature, humidity, and wind) on the measurement data may be bigger than structural damage, greatly limiting the efficiency and accuracy of the identification results [3]. In particular, temperature is considered as one of the most significant and influential environmental effects on civil structures [3]. The shifts of damage-sensitive features induced by temperature fluctuation are usually of the same order as those caused by acceptable levels of damage. Consequently, false diagnoses will occur if the temperature effects cannot be appropriately considered in the process of damage identification $[4,5]$. Hence, how to eliminate the detrimental effects of variable temperatures on damage identification is vital to reliable damage identification and requires further investigation.

Currently, two categories of vibration-based methods, signal-based methods and modal-based methods, have been extensively studied. The former group of methods relies on measured response data, while the latter one on modal parameters and their derivatives, such as frequencies, mode shapes, modal strain energy, and modal flexibility $[1,2,6]$. There are certain drawbacks in modal-based methods, and it is impossible to excite high modes at ambient conditions without high energy [6]. Errors in modal parameter identification and noise may inevitably make the change in 
damage-sensitive features indistinguishable as well. Therefore, modal-based methods usually have been proved to be inaccurate and unreliable for damage identification in reallife scenarios. The signal-based methods, depending on data from the measurements only, have been widely adopted in the literature for damage identification. To extract damagesensitive features from response data, time series analysis techniques have been widely utilized [1]. AR time series model-based approaches, in which damage indices such as Mahalanobis distance, residual error, and features are extracted from AR coefficients, have been used for damage diagnosis [7, 8]. Furthermore, ANN, one of the artificial intelligence and machine learning techniques, has been widely reported to eliminate operational and environmental effects in the process of damage diagnosis [8-10]. In recent years, combining the AR time series model and ANN methods for structural damage identification has garnered significant interests [7-9]. However, identifying damage locations and severities simultaneously is such a complex and time-consuming process such that it remains an enormous challenge under temperature variations so far.

In this paper, a damage identification approach, based on time series analysis in conjunction with two-step ANN models using acceleration responses under temperature variations, is explored. Aiming to compensate temperature effects, this two-step damage identification approach strives to accurately estimate a range of damage information such as damage location and severity simultaneously. Firstly, AR time series models are used to model the acceleration response data from the structure with and without damage at different temperatures. In addition, the damage-sensitive features are formulated based on AR coefficients. Then, two series of backpropagation neural network (BPNN) models, which utilize the combination of damage features and temperature variables as input data, are constructed to locate and subsequently quantify damage. Finally, numerical experiments of a four-story, steel-braced frame structure with different damage scenarios and temperatures are conducted to investigate the effectiveness and robustness of the proposed approach.

\section{Temperature Effects and Damage Identification}

2.1. Damage Identification under Varying Temperature Effects. In recent decades, research on robust damage identification techniques for civil structures under varying temperature conditions has gained momentum [11-16]. More recently, numerous machine learning techniques, such as support vector machine (SVM) [17], genetic algorithm (GA) [18], particle swarm optimization (PSO) [19], cuckoo search (CS) [20], and artificial neural network (ANN) [21-23], have been explored to eliminate temperature effects on vibration-based damage identification. Of these, ANN-based damage identification methods are the most extensively applied methods and have been increasingly receiving attention. Both $\mathrm{Gu}$ et al. [21] and Zhou et al. [24] proposed neural networkbased damage identification approaches, which utilize modal frequencies as damage-sensitive features, to alleviate temperature effects for structural damage identification. However, these ANN-based methods are usually plagued by drawbacks of complex ANN structures, large samples, and slow convergence rates. To achieve good generalization capability, hybrid ANN [25], ANN ensemble [9], and twostep ANN have been explored and adopted to recognize, locate, and quantify damage under temperature variations. Zhou et al. [24] successfully applied a two-step BPNNANNN approach to identify damage for the Ting Kau Bridge by using the long-term monitoring data; frequencies were first normalized to eliminate the temperature effects by using backpropagation neural network (BPNN) models, and damage was successfully identified by using the AANN model. The two-step ANN does not have the aforementioned issues. Nevertheless, two-step ANN methods mostly utilize modal parameters as the damage-sensitive features. Considering that modal parameter extraction and modal truncation inevitably yield accumulation of errors, minor damage will go undetected. Consequently, the efficiency and accuracy of these methods may be questionable for practical applications.

Time series analysis, which uses sequence of data points with uniform time intervals to create systems, can be applied to feature extraction, damage diagnosis, and damage classification without modal parameter identification in SHM [3]. Various time series models, such as $\operatorname{AR}[7,8,26], \mathrm{ARX}$ (autoregressive model with exogenous input) [27], and ARMA (autoregressive-moving average) [28], have been extensively explored, whose features based on their coefficients have been extracted as damage indices by the SHM community. Das et al. [1] conducted a comparative study of the effectiveness of different vibration-based damage identification methods and proved that time series analysis outperformed other methods in damage identification with the presence of operational or environmental nuisances. Furthermore, they have been successfully incorporated with SVM [29], hidden Markov model (HMM) [30], and ANNs $[7,22,23,31]$ to identify damage. To avoid false diagnoses, an approach combining sensor-clustering-based time-series analysis with the ANN, which was proposed by Kostić and Gül [22], could successfully determine the existence, location, and relative severity of damage for a footbridge finiteelement model under temperature variations.

As indicated above, a variety of ANNs and time series models can be employed to identify damage under varying temperature variations. Each method experiences some limitations. Some ANN-based methods need large samples and complex computation, while such problems do not exist when two-step ANN-based methods are applied. However, the effectiveness and accuracy of a majority of ANN-based methods may deteriorate due to modal identification errors. Although methods incorporating the time series model and ANNs show enormous potential for reliable damage identification, assessment of damage locations and severities simultaneously is difficult in the previous studies. Moreover, temperature data, which can be tracked reliably and easily nowadays, are usually neglected. Therefore, to reliably identify damage locations and severities simultaneously under varying temperature variations, the integration of 
time series analysis with two-step ANN models warrants further investigation.

\section{Methodologies}

3.1. The Autoregressive Time Series Model. Owing to its high sensitivity to damage features, the autoregressive (AR) time series method is extensively used to extract the damagesensitive features [8]. In a health monitoring system, a series of original response data can be obtained from sensors placed on various locations of structures with a certain sampling frequency. These data constitute the time series [7]. Assuming these observed time series data $\left\{x_{t},(t=1,2,3, \ldots, p)\right\}$ is a stationary, zero-mean stochastic process, another time series data $\left\{a_{t}\right\}$, which is a white noise sequence with a zero-mean value and a $\sigma_{a}^{2}$ variance, is given by

$$
x_{t}-\phi_{1} x_{t-1}-\phi_{2} x_{t-2}-\cdots-\phi_{p} x_{t-p}=a_{t},
$$

where $\phi_{j}(j=1,2,3, \ldots, p)$ and $P$ are referred to as the $j$ th autoregressive coefficients and the order of the AR model, respectively, $\phi_{i} x_{t-i}$ denotes the contribution (or influence) of the previous value at time $t-i$ to the current value at time $t$, and $\phi_{i}$ is the degree of such contribution (or influence); $\left\{x_{t}\right\}$, shown as equation (1), is the regression of its own past values, so it is termed as the autoregressive (AR) model.

In order to improve the modeling reliability, the initial observed data should be preprocessed. In this paper, after removing the abnormal data, the mean, and the trend, the normality and independence of those vibration data are checked. Subsequently, the attempts on order determination and parameter estimation should be made to define AR models for different sensor locations. Given that determining the optimal order is crucial for the establishment of time series models, Akaike's information criterion (AIC) is employed to determine the model order initially. Despite a higher-order model that can fit the dataset with high accuracy, it cannot be usually generalized to other datasets. On the contrary, a lower-order model cannot adequately characterize the dataset [8]. AIC, completely objective, is not influenced by subjective factors. Moreover, the optimal model with the smallest AIC value could be definitely chosen via this criterion, and AIC can be defined as follows:

$$
\mathrm{AIC}=N \log \sigma_{a}^{2}+2(p+1),
$$

where $N$ is the number of samples, $\sigma_{a}^{2}$ is the variance of the residual, and $p$ is the order of the model.

Secondly, parameter estimation of the AR model should be carried out. In recent years, two categories of methods, the direct estimation method and the recursive estimation method [32], have been employed. The former group of methods estimates the parameters by using the observed data or their statistical characteristics directly, such as the least square estimation method [33], least square estimation method based on autocorrelation coefficients, Yule-Walker solution method [34], and Ulrych-Clayton method [35]. The latter one mainly includes the parameter recursive estimation method, matrix recursive estimation method, and real- time recursive estimation method. The least square method, which is widely used among the direct methods, is employed in this paper due to its high accuracy and less computation, especially for linear models.

Finally, damage features, which are referred to as the most sensitive parameters varying with structural condition or other damage indicators, should be extracted. In this paper, the difference in the first three-order AR coefficients of the structure with and without damage is used as the damage feature, which can be written as

$$
\mathrm{DS}_{i}=\phi_{\text {if }}-\phi_{\text {id }}
$$

where $\mathrm{DS}_{i}$ is the damage feature of the $i$ th observed point, $\phi_{\text {if }}$ is the vector of the first three-order AR coefficients of the undamaged structure, and $\phi_{\text {id }}$ is the vector of the first threeorder AR coefficients of the damaged structure.

3.2. Backpropagation Neural Network. As a powerful tool of pattern recognition and classification [9], artificial neural network has increasingly received attention from structural dynamics researchers in the past. BPNN, a multilayer feedforward neural network, consists of an input layer, a hidden layer, and an output layer, as shown in Figure 1.

In essence, BPNN is a formulation used to operate the nonlinear principal component analysis (NLPCA) algorithm. After feeding two datasets $\mathbf{X}$ and $\mathbf{Y}$ with sufficient large data capacity into a BPNN model, dependencies among the two datasets will be extracted, and a nonlinear transformation mapping $\mathbf{X}$ into $\mathbf{Y}$ with minimum loss of information will be achieved. In the training phase, $X=\left\{\begin{array}{lllll}x_{1} & x_{2} & \ldots & x_{n}\end{array}\right\}^{T}$ is fed into the input layer with arbitrary small weight values firstly. Secondly, the outputs $\widehat{Y}$ will be obtained in the output layer, which are calculated using the following formula:

$$
y_{k}=h_{2}\left(\sum_{j=1}^{N_{2}} W_{j k}^{2} h_{1}\left(\sum_{i=1}^{N_{1}} W_{i j}^{1} x_{i}+b_{i}\right)+b_{j}\right),
$$

where $y_{i}$ and $x_{i}$ are the $i$ th components of $\widehat{Y}$ and $\mathbf{X}$, respectively, $W_{i j}^{k}$ is the weight connecting the $i$ th node in the $k$ th layer to the $j$ th node in the successive layer, $b_{j}$ is a node bias, $N_{i}$ is the number of nodes in each layer, and $h(x)$ is referred to as the transfer function, often a sigmoid transfer function in the hidden layer and linear transfer function in the output layer.

Finally, the error between the predicted outputs $\widehat{Y}$ and the target outputs $\mathbf{Y}$ is propagated in the inverse direction, and the weights and biases of each layer are adjusted. By iterating the aforementioned process until the error reaches a tolerable error, an appropriate BPNN model with good generalization capability, which can extract nonlinear correlations embedded in the two patterns, could be gained.

3.3. Damage Identification Flowchart under Varying Temperatures. Considering that temperature information of civil structures could be reliably and easily acquired recently, a BPNN-based damage identification approach using AR 


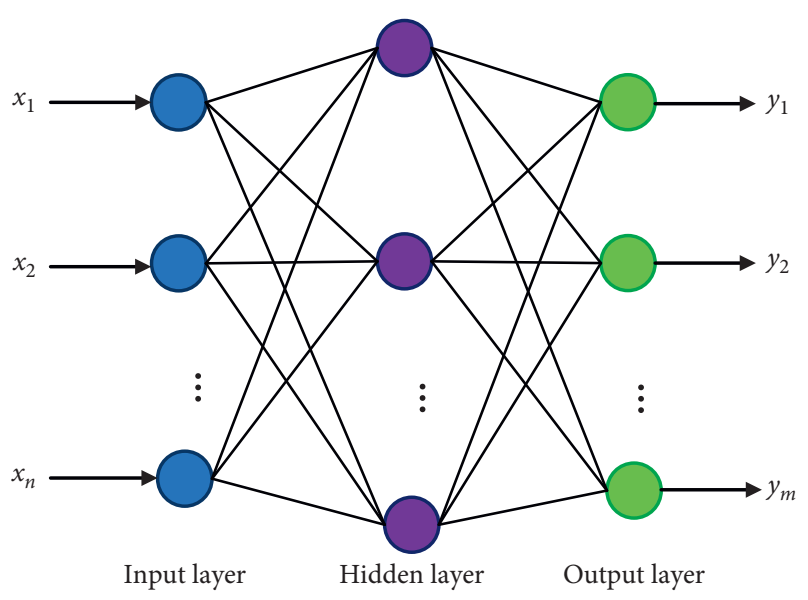

FIGURE 1: A schematic presentation of a backpropagation neural network (BPNN).

coefficients in conjunction with temperature variations is developed in this paper.

Figure 2 illustrates the procedure of this novel approach, which is described as follows:

(1) Obtain the acceleration responses of an undamaged structure excited by the Gauss white noise under temperature $T$.

(2) Fit the AR models to the acceleration signals using the method introduced, and acquire the first threeorder AR coefficients $\phi_{1, \text { if }}, \phi_{2, \text { if }}$, and $\phi_{3, \text { if }}$.

(3) To inflict damage on the structure by reducing the elastic modulus of elements, the severity of damage is defined as equation (5). In addition, to change the elastic modulus of the whole structure is to simulate temperature variations and extract the acceleration responses of the structure with different damage scenarios under temperature $T$.

$$
a_{j}=\left[1-\frac{(E)_{d}}{(E)_{u}}\right],
$$

where $(E)_{d}$ and $(E)_{u}$ are referred to as the elastic modulus of elements in damaged and intact states, respectively.

(4) Extract damage features $\mathrm{DS}_{1, i}, \mathrm{DS}_{2, i}$, and $\mathrm{DS}_{3, i}$ by using equation (3) after acquiring the AR model coefficients of the damaged structures $\phi_{1, d i}, \phi_{2, d i}$, and $\phi_{3, d i}$ by using the method introduced in Steps (1) and (2).

(5) Identify the damage location and severity under temperature variations based on two-step BPNN models. Initially, combine the damage feature extracted in Step (4) and temperature data as the new efficient input data of BPNN models, as shown in equation (6). BPNN models with the output vector including 0 and 1 , which denotes intact and damaged elements or damage patterns of the structure, are formulated to identify damage locations firstly. Then, another BPNN model with the output vector comprising the damage severity of the damaged elements, defined as equation (5), is built to quantify damage. In order to compensate detrimental temperature variations, datasets acquired from the structure in damaged and undamaged states under different temperatures are utilized to train these BPNN models.

$$
\begin{aligned}
\text { Input }= & \left\{T, \mathrm{DS}_{1,1}, \mathrm{DS}_{2,1}, \mathrm{DS}_{3,1}, \mathrm{DS}_{1,2}, \mathrm{DS}_{2,2},\right. \\
& \left.\mathrm{DS}_{3,2}, \ldots, \mathrm{DS}_{1, i}, \mathrm{DS}_{2, i}, \mathrm{DS}_{3, i}\right\},
\end{aligned}
$$

where $T$ represents the structural temperature, $\left\{\mathrm{DS}_{1, i} \mathrm{DS}_{2, i} \mathrm{DS}_{3, i}\right\}$ are referred to as the damage features at the $i$ th node.

\section{Simulation Experiment of a Frame Structure and Temperature Effect Analysis}

In order to verify the effectiveness and reliability of this damage identification approach in the presence of temperature variations, numerical impact experiments of a fourstory, two-bay $\times$ two-bay steel-braced frame, which is constructed by the ASCE (American Association of Civil Engineers) [36], are conducted.

4.1. ASCE Benchmark Structure and Numerical Simulation. As illustrated in Figure 3(a), the ASCE benchmark structure comprises 9 columns, 30 beams, and 24 braces, whose cross sections are $\mathrm{B} 100 \times 9, \mathrm{~S} 75 \times 11$, and $\mathrm{L} 25 \times 25 \times 3$, respectively. 16 floor slabs are uniformly distributed upon the beams, configuring a doubly symmetric four-story frame structure, $2.5 \mathrm{~m}$ long by $2.5 \mathrm{~m}$ wide [36]. Note that the weight of each slab placed on the first three-order floors is $1000 \mathrm{~kg}$, while that of the fourth floor is $750 \mathrm{~kg}$. Additionally, the connections between beams and columns are fixed, and the ends of diagonal braces are hinged [37]. A precise finite element (FE) model, as shown in Figure 3(b), is established to simulate the benchmark structure using SAP2000, and time history analyses are conducted. A nonlinear temperature-dependent elasticity modulus for steel, proposed by Yan et al. [38] and as illustrated in Figure 4, is defined to calculate the thermal effects. Additionally, damage is simulated by elastic modulus reduction of certain elements, resulting in a loss of stiffness. Acceleration-time histories of each node can be extracted via time history analyses. It should be noted that only braces of $Y$-plane1, as shown in Figure 5, are inflicted on the aforementioned damage.

The ASCE benchmark structure is excited at 15 and 29 nodes in the $Y$ direction by using the Gauss white noise with a frequency of $1000 \mathrm{~Hz}$ and a sampling time of $50 \mathrm{~s}$. Acceleration signals from nodes 5, 8, and 11 are collected for time-series modelling. The sampling frequency and duration of the datasets are $500 \mathrm{~Hz}$ and $20 \mathrm{~s}$, respectively. For conciseness, only the typical acceleration signal during the first 10 seconds from node 5 of the undamaged structure at $20^{\circ} \mathrm{C}$ is recorded. 5,000 consecutive data points from the 5th second to the 15 th second are selected from each node to fit the AR models. 


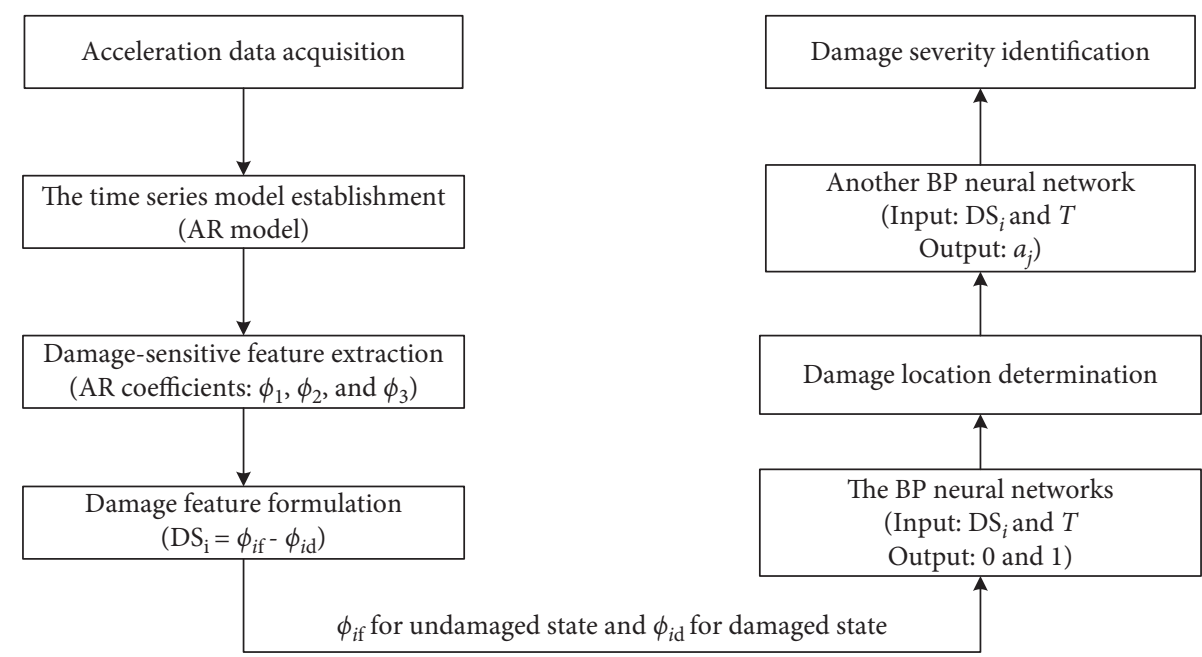

Figure 2: Flowchart of the proposed damage identification approach.

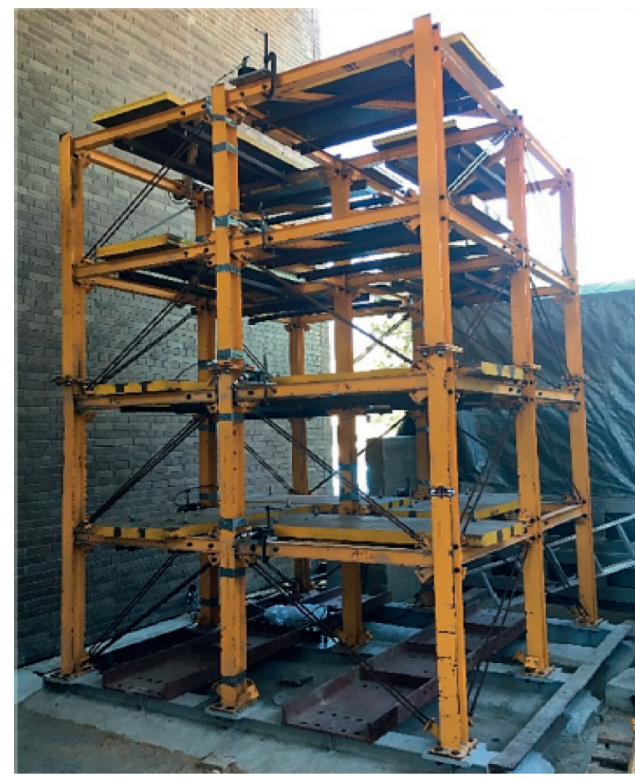

(a)

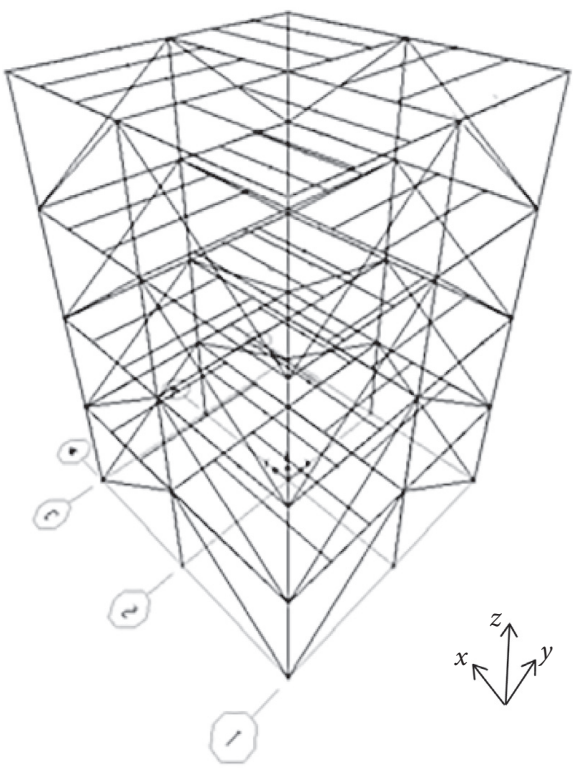

(b)

Figure 3: ASCE benchmark model: (a) steel-frame scale structure; (b) SAP2000 finite element model.

4.2. Analysis of the Time Series Model. Once the preprocessing of the initial acceleration data is completed, the optimal AR model orders and their coefficients are estimated for nodes 5,8 , and 11 by using the method introduced. AIC and the least square algorithm are employed to obtain the most suitable AR model by choosing the AR model order and estimating the coefficients. For the acceleration data obtained from node 5 of the undamaged ASCE benchmark structure at $20^{\circ} \mathrm{C}$, the relationship between AIC value and model order $p$ is illustrated in Figure 6. It is clearly shown that the AIC value decreases gradually with the increase of the order of the AR model and is negligible for AR order 10 and above. It can be seen that the optimal order is 47 with the minimal AIC value. Moreover, the AR (47) model yields a final prediction error (FPE) of $6.118 \times 10^{-6}$ and a mean square error (MSE) of $6.005 \times 10^{-6}$, indicating that the AR (47) model is suitable. Figure 7 shows the comparison of time histories of the input acceleration responses with the predicted responses corresponding to the AR (47) model. It can be observed that the predicted response of the AR (47) model almost coincides with the input response, verifying the aforementioned conclusion again. In addition, the details of the most suitable AR models for each observed node of the undamaged ASCE benchmark structure at $20^{\circ} \mathrm{C}$ and $30^{\circ} \mathrm{C}$ are listed in Table 1.

4.3. Damage and Temperature Effects on AR Model Coefficients (Feature Selection). The FE model established in the previous section is used to simulate structural damage and 


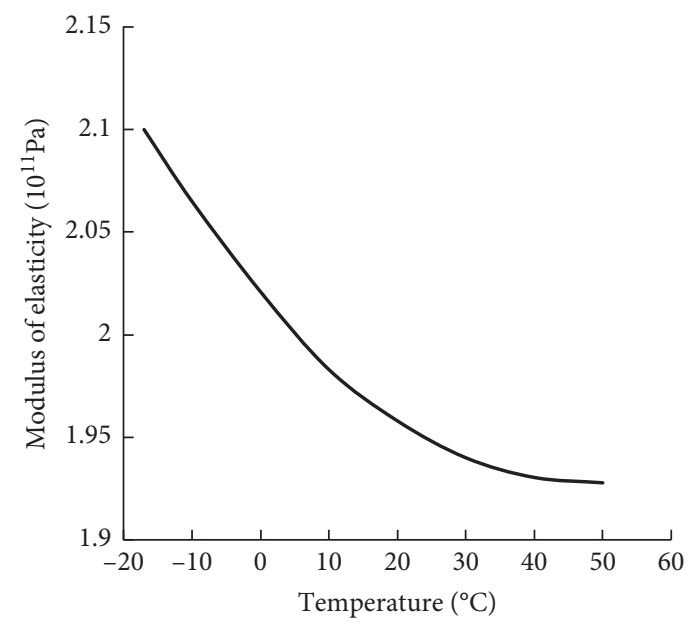

FIGURE 4: The relationship between elastic modulus of steel and temperature [38].

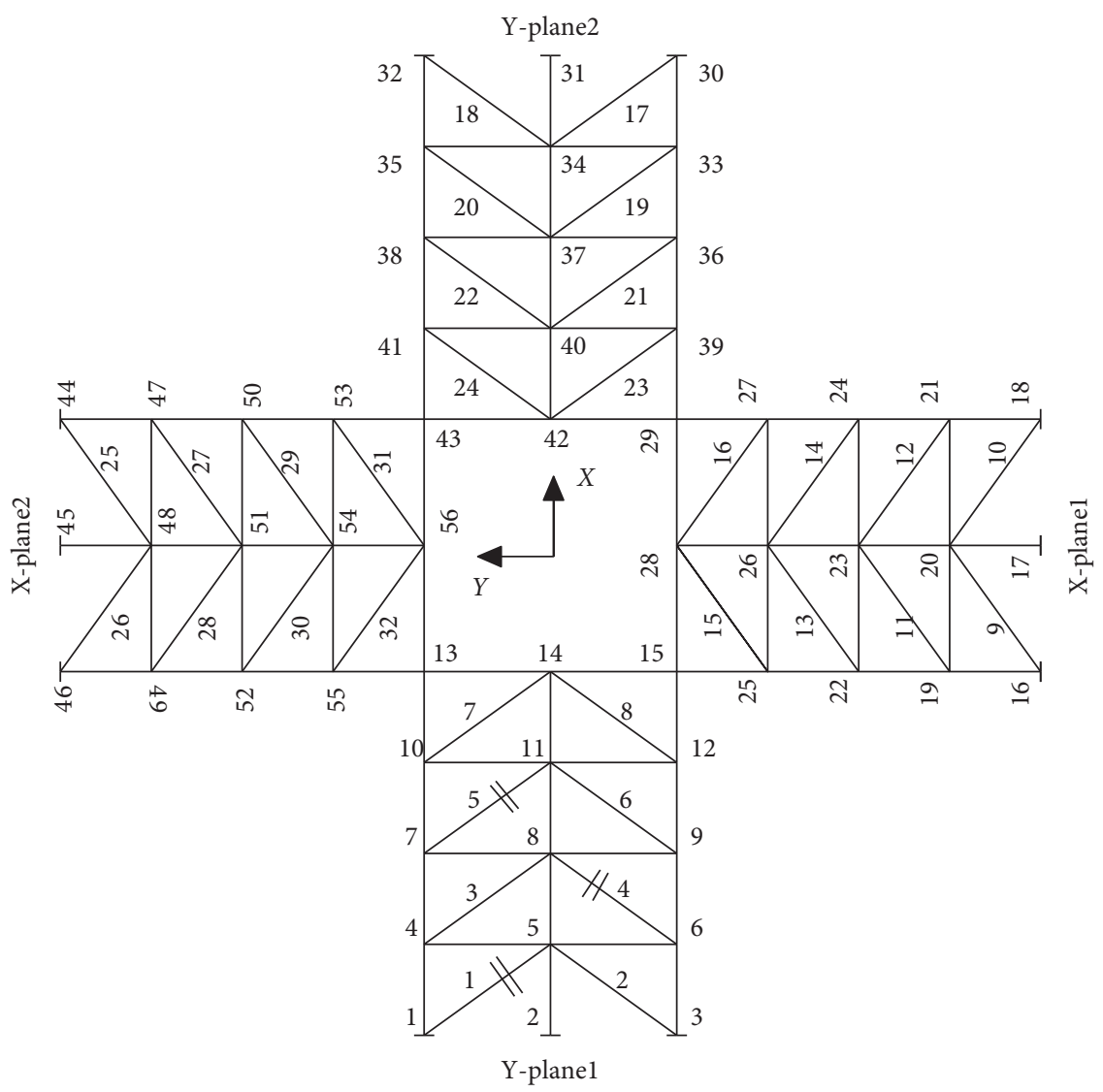

Figure 5: Diagram of the element and node number.

analyse the temperature effects on AR model coefficients. Damage is simulated by reducing the elastic modulus of brace 1 , and $a_{j}$ (see equation (5)) is $40 \%$. The temperature of the whole structure varies from $-10^{\circ} \mathrm{C}$ to $40^{\circ} \mathrm{C}$, which is simulated by changing the elasticity modulus of steel according to Figure 4 . After a series of time history analyses, the most suitable AR models are established to fit the response data from the three observed nodes.

Then, the first three-order AR coefficients for nodes 5, 8, and 11 are extracted, whose distribution is shown in Figure 8 when the temperature changes from $-15^{\circ} \mathrm{C}$ to $40^{\circ} \mathrm{C}$. It is clearly shown that the first three-order $A R$ coefficients 


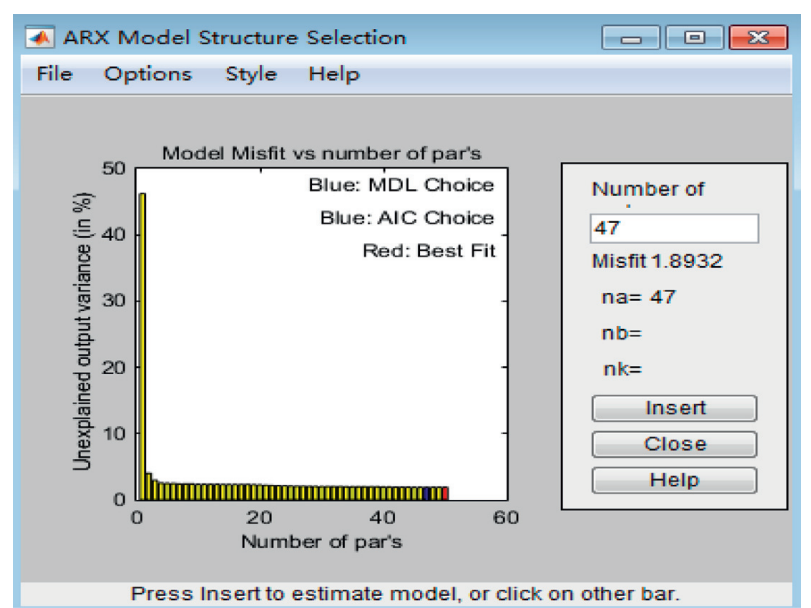

FIguRE 6: Variation of AIC with model order for node 5 of the undamaged ASCE benchmark structure at $20^{\circ} \mathrm{C}$.

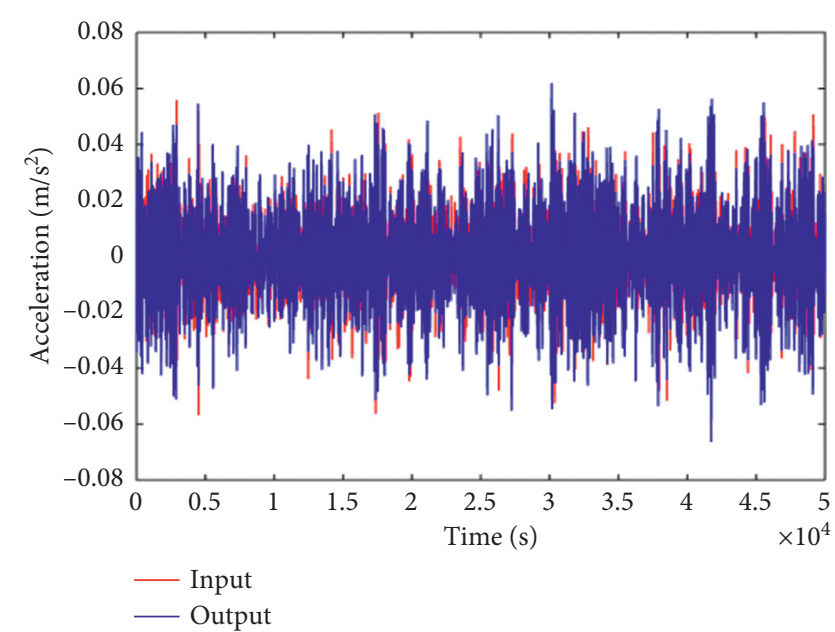

FIgURE 7: The acceleration signal of the AR model input and predicted output.

TABLE 1: The most suitable AR models for each node of the undamaged ASCE benchmark structure under $20^{\circ} \mathrm{C}$ and $30^{\circ} \mathrm{C}$.

\begin{tabular}{cccccc}
\hline$T\left({ }^{\circ} \mathrm{C}\right)$ & Node number & Order & $\phi_{1}$ & $\phi_{2}$ & $\phi_{3}$ \\
\hline \multirow{3}{*}{20} & 5 & 47 & -1.918 & 2.047 & -0.7244 \\
& 8 & 46 & -2.481 & 3.082 & -1.821 \\
& 11 & 47 & -1.575 & 1.312 & -0.2856 \\
30 & 5 & 47 & -1.919 & 2.044 & -0.7213 \\
& 8 & 46 & -2.481 & 3.077 & -1.813 \\
& 11 & 47 & -1.576 & 1.311 & -0.2858 \\
\hline
\end{tabular}

almost remain stable under varying temperatures, while the third-order AR coefficients occur steady, visible change. Among them, the third-order coefficient for node 5 yields the maximum variance ratio, which reaches $5.48 \%$.

By contrast, the other scenarios are investigated, the temperature of the whole structure remains $20^{\circ} \mathrm{C}$, and $a_{j}$ of brace 1, which denotes damage severity, varies from 0 to $100 \%$ with an interval of $10 \%$. Figure 9 shows the distribution of the first three-order AR coefficients for nodes 5,8 , and 11 of the damaged structure with different severities. It is seen that each coefficient for all the observed nodes shows considerable shifts, and the maximum variance ratio is up to $60.06 \%$. With increasing of damage severity, the first three-order AR coefficients for node 5 present down, up, and down trends, respectively. The variation law of those coefficients for node 11 is the same, while that for node 8 is inverse. Furthermore, local minor damage causes obvious shifts of AR coefficients for the structure with constant temperature, indicating high sensitivity of those AR coefficients to structural damage.

In summary, the first three-order AR coefficients are more sensitive to damage occurring in structures than temperature fluctuations. Thus, they can be adopted as damage-sensitive features for damage identification. However, owing to the existence of unavoidable cases, the changes caused by temperature fluctuations can even mask those induced by damage. Therefore, if temperature variations are not taken into account for damage identification based on time series models, false diagnosis will occur.

\section{Damage Identification}

In this section, the proposed method, which integrates the time series model with the BPNN, is employed to identify damage of the ASCE steel-braced frame structure under varying temperatures. After fitting the AR time series models to the response data, the difference between the first threeorder AR coefficients from damaged and undamaged structures is extracted as damage features, which are fed into BPNN ensemble with the corresponding temperature values $(T)$ to identify the locations and severities of damage. In this process, the robustness of this novel approach is tested in order to avoid false diagnosis. Furthermore, its effectiveness is also examined by considering noise.

5.1. Damage Identification under Varying Temperatures. To test the robustness of the proposed method, damage scenarios are assumed and listed in Table 2. Two kinds of damage, single and multiple damage, are inflicted on braces 1,4 , and 5 by reducing their elastic modulus, constructing 6 damage cases. Moreover, the $a_{j}$ value, defined in equation (5) to quantify damage severity, is $40 \%, 50 \%, 60 \%, 70 \%$, or $80 \%$, and the value of temperature $(T)$ is $20^{\circ} \mathrm{C}$ or $30^{\circ} \mathrm{C}$. As a result, 60 FE models are created for the 6 aforementioned damage cases. After the time history analysis, the acceleration signals obtained from nodes 5, 8, and 11 are modelled as AR time series. The differences between the first three-order AR coefficients of those nodes from the damaged and undamaged structures are extracted as damage features. The integration of these damage features with temperature values is used as the input data of BPNN models. To identify the location and severity of structural damages, a neural network ensemble, which combines two individual BPNN models, is formulated by using the same inputs. The output vector of one BPNN model is the indictor of damaged braces or damage patterns, of which the other BPNN model is the 


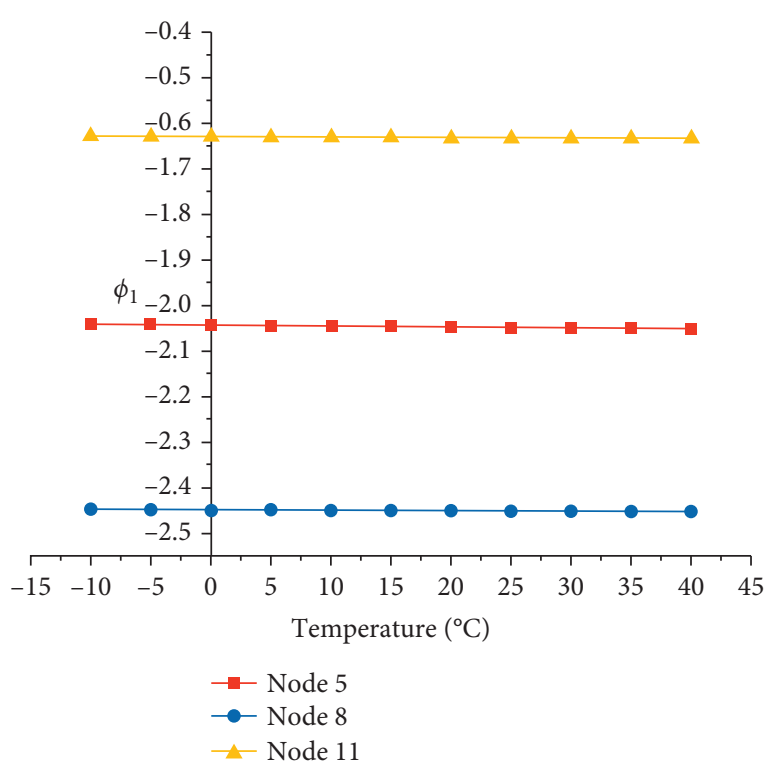

(a)

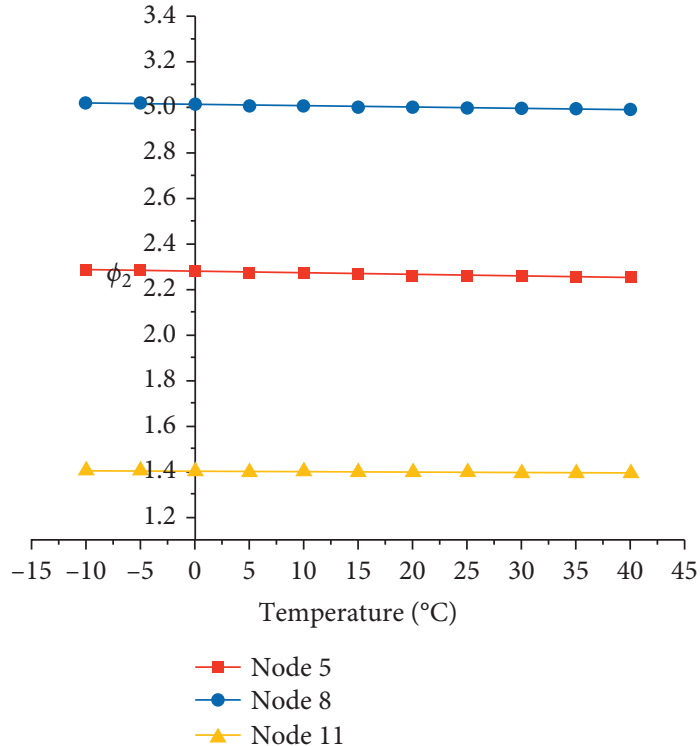

(b)

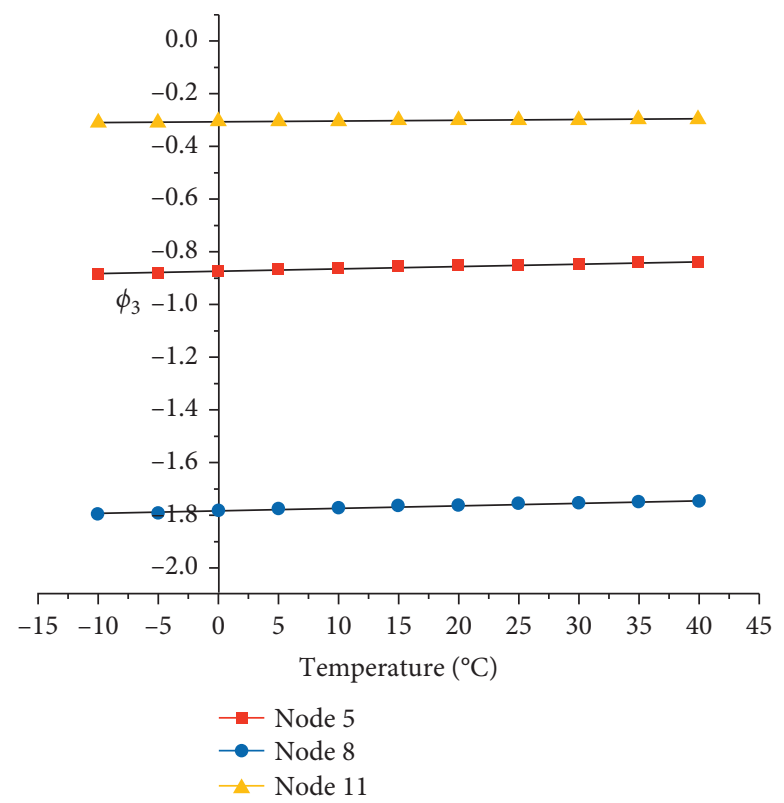

(c)

FIGURE 8: The relationship between the first three-order AR coefficients and temperature: (a) first-order AR coefficients; (b) second-order AR coefficients; (c) third-order AR coefficients.

indicator of damage extent consisting of the $a_{j}$ values of braces.

In this paper, the dataset from all the aforementioned damage scenarios, which comprises 60 observations, is divided into two parts, a training set containing 36 observations and a testing set containing 24 observations; and the data from single and multiple damage states account for half of each set. More specifically, data from the steel-braced frame structure with the $40 \%, 60 \%$, and $80 \% a_{j}$ values comprise the training set, and the remaining data comprise the testing set.
The number of neurons of input layers represents the number of damage features as input data, and the number of neurons for hidden layers is determined via trial training. The output layer of each network consists of nodes indicating the damage locations or damage severities of damaged braces denoted by $a_{j}$ in equation (5). The optimal number of hidden layer nodes of the two BPNN models is 14 by trial and error. Thus, a 10-14-3 BPNN model is formulated to identify damage locations, while a 10-14-1 or 10-14-2 BPNN model is configured to identify severities of single or multiple damage states in this paper. For the first BPNN model, 


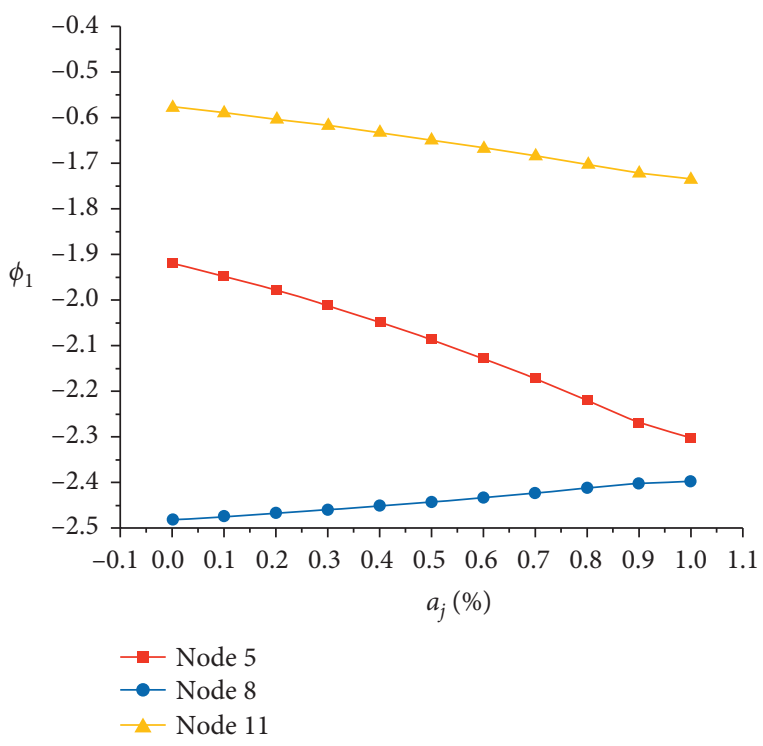

(a)

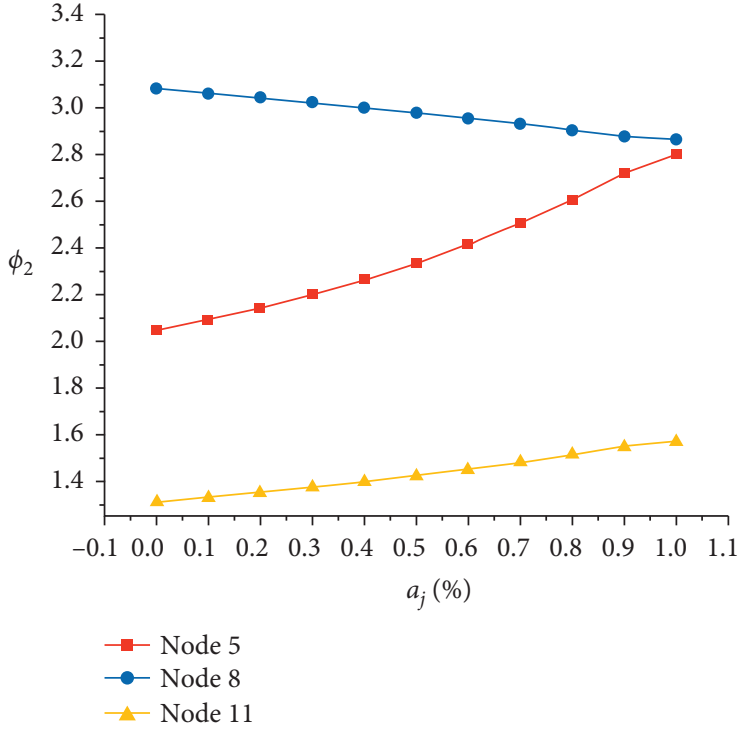

(b)

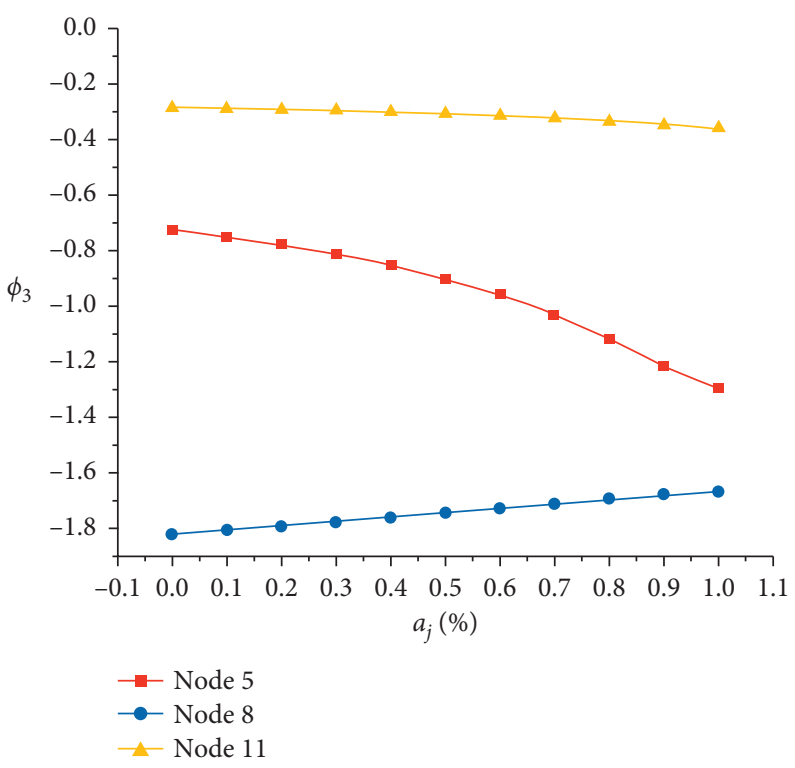

(c)

FIGURE 9: The relationship between the first three-order AR coefficients and damage severity: (a) first-order AR coefficients; (b) secondorder AR coefficients; (c) third-order AR coefficients.

TABLE 2: Numerical damage scenarios of the ASCE steel-braced frame structure.

\begin{tabular}{lcc}
\hline Type & Damage scenarios & Damage location \\
\hline \multirow{3}{*}{ Single } & DC 1 & Brace 1 \\
& DC 2 & Brace 4 \\
\multirow{3}{*}{ Multiple } & DC 3 & Brace 5 \\
& DC 4 & Braces 1,4 \\
& DC 5 & Braces 1,5 \\
& DC 6 & Braces 4,5 \\
\hline
\end{tabular}

both transfer functions of the hidden layer and output layer are tan-sigmoid. For the second BPNN model, their transfer functions are tan-sigmoid and pureline, respectively.
After adequate training using the LM (Levenberg-Marquardt) algorithm with different random initializations, the testing data are fed into the trained BPNN models to test the effects of the proposed approach. The identification results of damage locations and severities for the single damage cases are listed in Tables 3 and 4, respectively. It should be noted that the three output nodes of the first BPNN model indicate the status of braces 1, 4, and 5 . Additionally, 0 denotes undamaged, and 1 denotes damaged. For conciseness, the results of the training set are omitted in those tables. As presented in Table 3, the outputs of the first BPNN model are almost identical with the target outputs, indicating that the proposed approach can accurately identify the damaged brace for the single damage case 
TABLE 3: The identification results of damage locations when one brace is damaged.

\begin{tabular}{|c|c|c|c|c|c|}
\hline \multirow{2}{*}{ Damage scenarios } & \multirow{2}{*}{$a_{j}(\%)$} & \multirow{2}{*}{$T\left({ }^{\circ} \mathrm{C}\right)$} & \multicolumn{3}{|c|}{ Output nodes } \\
\hline & & & First & Second & Third \\
\hline \multirow{4}{*}{ DC 1} & \multirow{2}{*}{50} & 20 & $0.99999(1)$ & $3.61 \times 10^{-7}(0)$ & $7.28 \times 10^{-5}(0)$ \\
\hline & & 30 & $1(1)$ & $1.53 \times 10^{-7}(0)$ & $8.54 \times 10^{-8}(0)$ \\
\hline & \multirow{2}{*}{70} & 20 & $1(1)$ & $2.51 \times 10^{-5}(0)$ & $1.15 \times 10^{-8}(0)$ \\
\hline & & 30 & $0.999839(1)$ & $5.62 \times 10^{-6}(0)$ & $1.29 \times 10^{-5}(0)$ \\
\hline \multirow{4}{*}{ DC 2} & \multirow{2}{*}{50} & 20 & $8.25 \times 10^{-6}(0)$ & $0.999981(1)$ & $6.41 \times 10^{-7}(0)$ \\
\hline & & 30 & $5.15 \times 10^{-7}(0)$ & 0.999943 (1) & $8.18 \times 10^{-5}(0)$ \\
\hline & \multirow{2}{*}{70} & 20 & $1.12 \times 10^{-6}(0)$ & $0.999978(1)$ & $1.22 \times 10^{-5}(0)$ \\
\hline & & 30 & $1.11 \times 10^{-5}(0)$ & $0.999934(1)$ & $9.95 \times 10^{-6}(0)$ \\
\hline \multirow{4}{*}{ DC 3} & \multirow{2}{*}{50} & 20 & $8.05 \times 10^{-5}(0)$ & $6.69 \times 10^{-6}(0)$ & $0.999779(1)$ \\
\hline & & 30 & $8.82 \times 10^{-5}(0)$ & $1.21 \times 10^{-6}(0)$ & $0.999833(1)$ \\
\hline & \multirow[b]{2}{*}{70} & 20 & $3.34 \times 10^{-5}(0)$ & $5.95 \times 10^{-6}(0)$ & $0.99993(1)$ \\
\hline & & 30 & $6.89 \times 10^{-5}(0)$ & $4.26 \times 10^{-7}(0)$ & $0.999921(1)$ \\
\hline
\end{tabular}

The target results of networks are shown in parentheses.

TABle 4: The identification results of damage severities when one brace is damaged.

\begin{tabular}{|c|c|c|c|c|c|}
\hline Damaged brace & $a_{j}(\%)$ & $T\left({ }^{\circ} \mathrm{C}\right)$ & Target output & Actual output & Relative error (\%) \\
\hline \multirow{4}{*}{1} & \multirow{2}{*}{50} & 20 & 0.5 & 0.485 & -2.99 \\
\hline & & 30 & 0.5 & 0.507 & 1.46 \\
\hline & \multirow{2}{*}{70} & 20 & 0.7 & 0.728 & 3.99 \\
\hline & & 30 & 0.7 & 0.757 & 8.11 \\
\hline \multirow{4}{*}{4} & \multirow{2}{*}{50} & 20 & 0.5 & 0.500 & 0.09 \\
\hline & & 30 & 0.5 & 0.519 & 3.89 \\
\hline & \multirow{2}{*}{70} & 20 & 0.7 & 0.742 & 6.04 \\
\hline & & 30 & 0.7 & 0.779 & 11.31 \\
\hline \multirow{4}{*}{5} & \multirow{2}{*}{50} & 20 & 0.5 & 0.455 & -8.91 \\
\hline & & 30 & 0.5 & 0.457 & -8.63 \\
\hline & \multirow{2}{*}{70} & 20 & 0.7 & 0.734 & 4.87 \\
\hline & & 30 & 0.7 & 0.718 & 2.57 \\
\hline
\end{tabular}

regardless of varying temperatures. From Table 4, the relative error of the damage severities between the BPNN model outputs and the target outputs is mainly within $6 \%$. Only a few relative errors exceed $6 \%$ with a maximum value of $11.31 \%$. However, this error is allowable for damage diagnosis.

The two-step BPNN models are sufficiently trained by using the training data, and the target outputs of the first BPNN model represent damage patterns of multiple damage cases listed in Table 5. Tables 6 and 7 show the identification results produced by the trained BPNN models. Likewise, damage locations (output nodes of the first BPNN model) can be identified, and their severities (output nodes of the second BPNN model) are predicted accurately for all multiple damage cases (DC 4, DC 5, and DC 6) even though the maximum value of the relative error of damage severities between the BPNN model outputs and the target outputs reaches $12.33 \%$. From these results, it is found that the twostep BPNN-based damage identification is efficient and accurate for assessing damages under varying temperatures.

5.2. Damage Identification with Noise-Contaminated Data. In the real-life scenario, various factors, such as environmental noise and measurement errors, inevitably contaminate the measured data. It is believed that the presence of
TABLE 5: Target outputs of the first BPNN model and the corresponding damage patterns for multiple damage cases.

\begin{tabular}{lccc}
\hline Damage braces & 1,4 & 1,5 & 4,5 \\
\hline 1,4 & 1 & 0 & 0 \\
1,5 & 0 & 1 & 0 \\
4,5 & 0 & 0 & 1 \\
\hline
\end{tabular}

noise may interfere with the damage identification data, hence causing false diagnoses [4]. To check the robustness of the proposed method, noises should be added to the acceleration signal to simulate the real situation. In this paper, the noise-contaminated acceleration data are defined as

$$
a_{z i}=a_{i}+a_{i \max } \cdot \operatorname{randn} \cdot n_{s}
$$

where $a_{i}$ and $a_{z i}$ are referred to as noise-free acceleration data and noise-contaminated acceleration data from the measuring point $i$, respectively, randn denotes the white Gaussian noise with zero mean and 1 standard deviation, and $n_{s}$ is the noise intensity.

Different noise contamination levels (from 1\% to 6\%) are added to the noise-free acceleration data from the undamaged ASCE benchmark structure at $20^{\circ} \mathrm{C}$, and $\mathrm{AR}$ models are built.

To compare the first three-order AR coefficients of these noise-corrupted data and the noise-free data from 
TABLE 6: The identification results of damage locations when two braces are damaged.

\begin{tabular}{|c|c|c|c|c|c|}
\hline \multirow{2}{*}{ Damage scenarios } & \multirow{2}{*}{$a_{j}(\%)$} & \multirow{2}{*}{$T\left({ }^{\circ} \mathrm{C}\right)$} & \multicolumn{3}{|c|}{ Output nodes } \\
\hline & & & First & Second & Third \\
\hline \multirow{4}{*}{ DC 4} & \multirow{2}{*}{50} & 20 & $1(1)$ & $3.66 \times 10^{-8}(0)$ & $8.99 \times 10^{-9}(0)$ \\
\hline & & 30 & $1(1)$ & $1.32 \times 10^{-8}(0)$ & $4.68 \times 10^{-10}(0)$ \\
\hline & \multirow{2}{*}{70} & 20 & $1(1)$ & $7.08 \times 10^{-7}(0)$ & $4.58 \times 10^{-9}(0)$ \\
\hline & & 30 & $1(1)$ & $3.51 \times 10^{-7}(0)$ & $1.04 \times 10^{-8}(0)$ \\
\hline \multirow{4}{*}{ DC 5} & \multirow{2}{*}{50} & 20 & $1.03 \times 10^{-5}(0)$ & $0.99857(1)$ & $1.93 \times 10^{-5}(0)$ \\
\hline & & 30 & $5.61 \times 10^{-7}(0)$ & $0.9816(1)$ & $0.010006(0)$ \\
\hline & \multirow[b]{2}{*}{70} & 20 & $3.84 \times 10^{-8}(0)$ & $1(1)$ & $1.25 \times 10^{-6}(0)$ \\
\hline & & 30 & $3.02 \times 10^{-7}(0)$ & $1(1)$ & $1.35 \times 10^{-6}(0)$ \\
\hline \multirow{4}{*}{ DC 6} & \multirow{2}{*}{50} & 20 & $5.31 \times 10^{-5}(0)$ & $4.95 \times 10^{-7}(0)$ & 0.99953 (1) \\
\hline & & 30 & $1.41 \times 10^{-4}(0)$ & $2.66 \times 10^{-7}(0)$ & $0.99957(1)$ \\
\hline & \multirow{2}{*}{70} & 20 & $7.44 \times 10^{-7}(0)$ & $1.84 \times 10^{-6}(0)$ & $0.99999(1)$ \\
\hline & & 30 & $4.98 \times 10^{-7}(0)$ & $7.60 \times 10^{-7}(0)$ & $0.99999(1)$ \\
\hline
\end{tabular}

The target results of networks are shown in the parentheses.

TABLE 7: The identification results of damage severities when two braces are damaged.

\begin{tabular}{|c|c|c|c|c|c|c|c|c|}
\hline \multirow[t]{2}{*}{ Damaged braces } & \multirow{3}{*}{$\begin{array}{c}a_{j}(\%) \\
50\end{array}$} & \multirow{2}{*}{$\frac{T\left({ }^{\circ} \mathrm{C}\right)}{20}$} & \multicolumn{2}{|c|}{ Target output } & \multicolumn{2}{|c|}{ Actual output } & \multicolumn{2}{|c|}{ Relative error (\%) } \\
\hline & & & 0.5 & 0.5 & 0.47851 & 0.47503 & 4.30 & 4.99 \\
\hline \multirow{3}{*}{1,4} & & 30 & 0.5 & 0.5 & 0.47236 & 0.46795 & 5.53 & 6.41 \\
\hline & & 20 & 0.7 & 0.7 & 0.75088 & 0.75377 & -7.27 & -7.68 \\
\hline & 70 & 30 & 0.7 & 0.7 & 0.76326 & 0.74738 & -9.04 & -6.77 \\
\hline \multirow{4}{*}{1,5} & & 20 & 0.5 & 0.5 & 0.43922 & 0.43835 & 12.16 & 12.33 \\
\hline & 50 & 30 & 0.5 & 0.5 & 0.45076 & 0.45346 & 9.85 & 9.31 \\
\hline & 70 & 20 & 0.7 & 0.7 & 0.76629 & 0.76308 & -9.47 & -9.01 \\
\hline & 70 & 30 & 0.7 & 0.7 & 0.74215 & 0.74138 & -6.02 & -5.91 \\
\hline \multirow{4}{*}{4,5} & & 20 & 0.5 & 0.5 & 0.48209 & 0.48442 & 3.58 & 3.12 \\
\hline & 50 & 30 & 0.5 & 0.5 & 0.47271 & 0.4785 & 5.46 & 4.30 \\
\hline & 70 & 20 & 0.7 & 0.7 & 0.77159 & 0.7673 & -10.23 & -9.61 \\
\hline & 70 & 30 & 0.7 & 0.7 & 0.76966 & 0.76332 & -9.95 & -9.05 \\
\hline
\end{tabular}

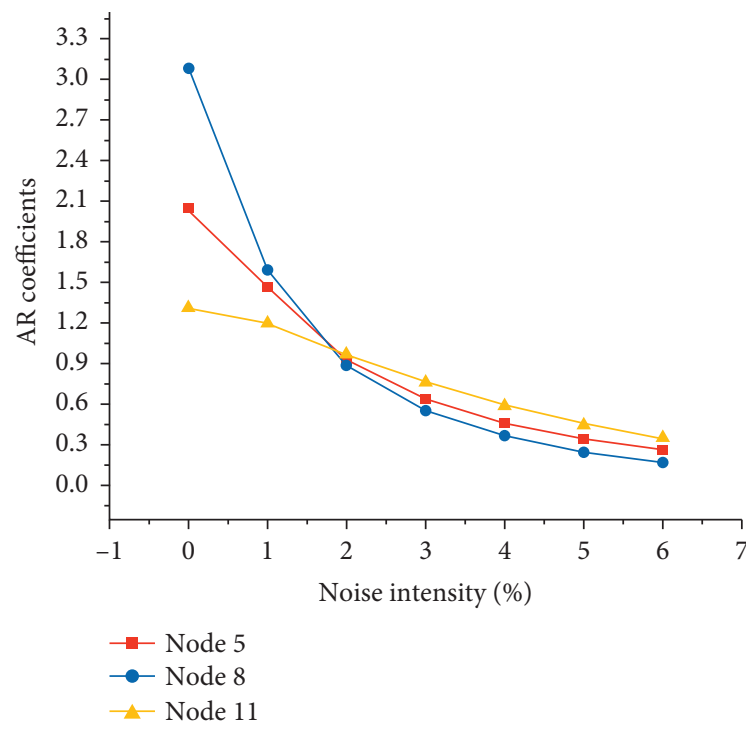

(a)

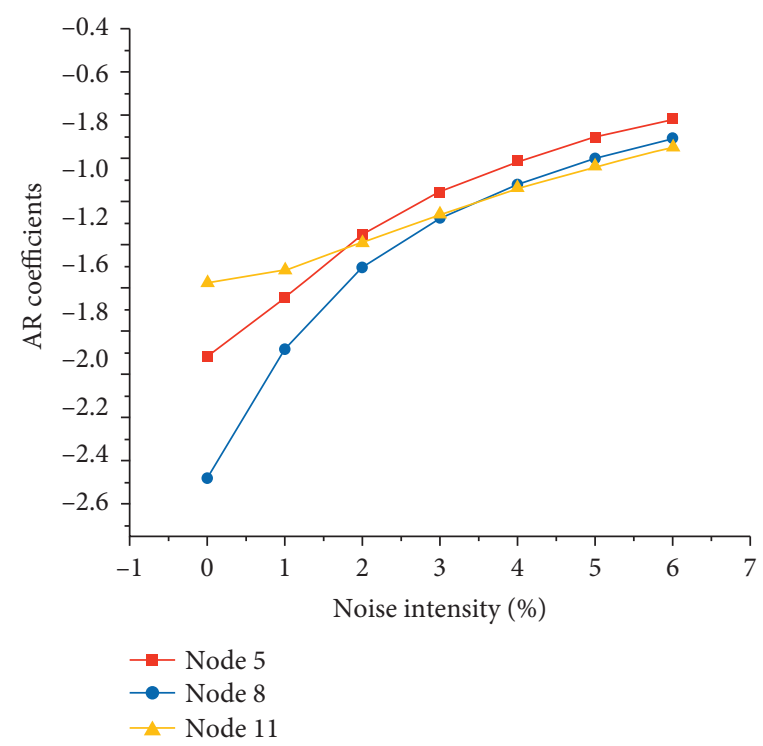

(b)

Figure 10: Continued. 


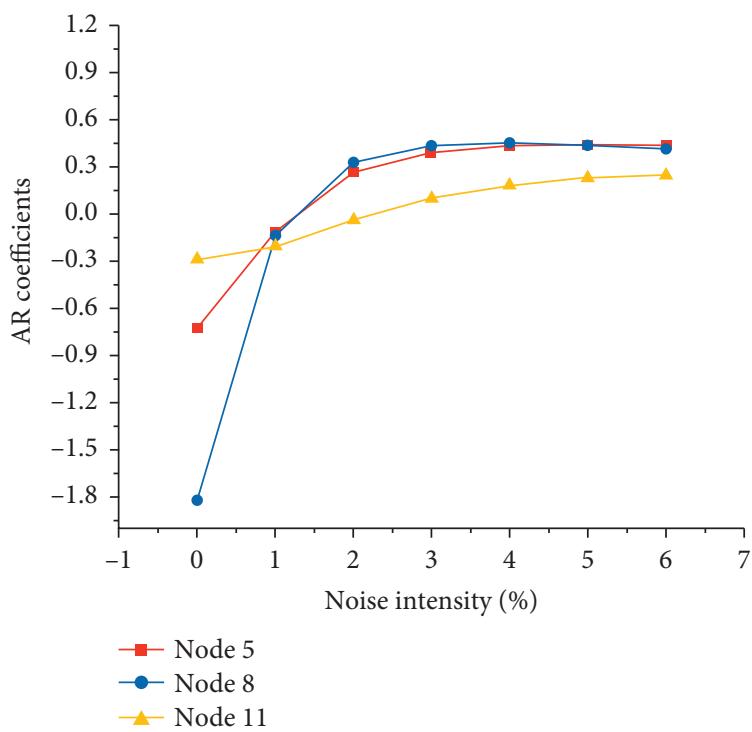

(c)

FIgURE 10: Comparison of the first three-order AR coefficients of noise-corrupted data and noise-free data: (a) first-order AR coefficients; (b) second-order AR coefficients; (c) third-order AR coefficients.

TABLe 8: The identification results of damage locations when one brace is damaged with noises.

\begin{tabular}{|c|c|c|c|c|c|c|c|c|}
\hline \multirow{3}{*}{ Damage scenarios } & \multirow{3}{*}{$a_{j}(\%)$} & \multirow{3}{*}{$T\left({ }^{\circ} \mathrm{C}\right)$} & \multicolumn{6}{|c|}{ Output nodes } \\
\hline & & & \multicolumn{3}{|c|}{ Noise intensity (1\%) } & \multicolumn{3}{|c|}{ Noise intensity (3\%) } \\
\hline & & & First & Second & Third & First & Second & Third \\
\hline \multirow{4}{*}{ DC 1} & \multirow[b]{2}{*}{50} & 20 & $0.999984(1)$ & $1.28 \times 10^{-5}(0)$ & $3.41 \times 10^{-6}(0)$ & $1(1)$ & $2.93 \times 10^{-7}(0)$ & $1.69 \times 10^{-9}(0)$ \\
\hline & & 30 & $0.999993(1)$ & $1.28 \times 10^{-5}(0)$ & $2.26 \times 10^{-6}(0)$ & $1(1)$ & $1.49 \times 10^{-7}(0)$ & $4.25 \times 10^{-10}(0)$ \\
\hline & \multirow{2}{*}{70} & 20 & $0.999994(1)$ & $5.19 \times 10^{-6}(0)$ & $2.11 \times 10^{-6}(0)$ & $1(1)$ & $1.50 \times 10^{-7}(0)$ & $1.15 \times 10^{-12}(0)$ \\
\hline & & 30 & $0.999977(1)$ & $0.000123(0)$ & $2.08 \times 10^{-7}(0)$ & $1(1)$ & $5.30 \times 10^{-7}(0)$ & $2.79 \times 10^{-13}(0)$ \\
\hline \multirow{4}{*}{ DC 2} & \multirow{2}{*}{50} & 20 & $4.04 \times 10^{-6}(0)$ & $0.999999(1)$ & $4.86 \times 10^{-6}(0)$ & $5.60 \times 10^{-5}(0)$ & $0.999972(1)$ & $7.43 \times 10^{-8}(0)$ \\
\hline & & 30 & $2.15 \times 10^{-5}(0)$ & $0.999999(1)$ & $1.42 \times 10^{-6}(0)$ & $9.15 \times 10^{-4}(0)$ & $0.999981(1)$ & $1.21 \times 10^{-8}(0)$ \\
\hline & \multirow[b]{2}{*}{70} & 20 & $1.79 \times 10^{-5}(0)$ & $1(1)$ & $8.00 \times 10^{-7}(0)$ & $2.02 \times 10^{-6}(0)$ & $0.999999(1)$ & $1.04 \times 10^{-6}(0)$ \\
\hline & & 30 & $4.59 \times 10^{-5}(0)$ & $0.999999(1)$ & $3.82 \times 10^{-7}(0)$ & $4.20 \times 10^{-6}(0)$ & $1(1)$ & $1.40 \times 10^{-6}(0)$ \\
\hline \multirow{4}{*}{ DC 3} & \multirow{2}{*}{50} & 20 & $2.22 \times 10^{-5}(0)$ & $1.34 \times 10^{-5}(0)$ & $0.999981(1)$ & $1.56 \times 10^{-6}(0)$ & $4.04 \times 10^{-7}(0)$ & $0.999997(1)$ \\
\hline & & 30 & $2.29 \times 10^{-5}(0)$ & $1.24 \times 10^{-5}(0)$ & $0.999984(1)$ & $1.67 \times 10^{-6}(0)$ & $1.69 \times 10^{-6}(0)$ & $1(1)$ \\
\hline & \multirow{2}{*}{70} & 20 & $5.42 \times 10^{-6}(0)$ & $4.16 \times 10^{-5}(0)$ & $0.999994(1)$ & $8.70 \times 10^{-7}(0)$ & $1.10 \times 10^{-6}(0)$ & 0.999998 (1) \\
\hline & & 30 & $2.61 \times 10^{-5}(0)$ & $1.53 \times 10^{-5}(0)$ & $0.999982(1)$ & $6.36 \times 10^{-7}(0)$ & $3.88 \times 10^{-6}(0)$ & $1(1)$ \\
\hline
\end{tabular}

The target results of networks are shown in the parentheses.

observation nodes 5,8 , and 11 , the relation curves of the first three-order AR coefficients and noise intensity are obtained, as shown in Figure 10. It is evident that the noise interference results in notable changes to all AR coefficients. Moreover, there is a tendency for those AR coefficient variations to increase with the intensity of noise. Therefore, it is necessary to investigate the antinoise ability of the damage identification method based on time series and neural network under varying temperatures.

Two different noise contamination levels (1\% and 3\%) are applied to acceleration data from the ASCE benchmark structure in the healthy state, and all damage scenarios are illustrated in Table 2. Damage features are extracted, and the training and testing datasets are acquired by using the same process expounded in former discussion. Then, two-step BPNN models are formulated, trained, and tested with those noise-corrupted data. For the sake of brevity, only the identification results for the testing data are given. As observed from Table 8, the output of the first BPNN models for single damaged brace is close to 1 and that of other braces is close to 0 . Table 9 shows that the accuracy of damage identification decreases as the noise level increases, and the maximum value of the relative error is up to $16.94 \%$ for a noise level of $3 \%$. Nevertheless, the average relative errors of all the single damage cases are $5.26 \%$ and $7.32 \%$ corresponding to $1 \%$ and $3 \%$ noise pollution levels, which indicate that the second BPNN model is capable of identifying the severities of all single damage cases with high accuracy.

For the multiple damage scenarios, damage identification results are depicted in Tables 10 and 11. The outcomes of damage localization listed in Table 10 for $1 \%$ and $3 \%$ noise levels are precise for all damage scenarios. As it is observed 
TABle 9: The identification results of damage severities when one brace is damaged with noises.

\begin{tabular}{|c|c|c|c|c|c|c|c|}
\hline \multirow{2}{*}{ Damaged brace } & \multirow{2}{*}{$a_{j}(\%)$} & \multirow{2}{*}{$T\left({ }^{\circ} \mathrm{C}\right)$} & \multirow{2}{*}{ Target output } & \multicolumn{2}{|c|}{ Noise intensity (1\%) } & \multicolumn{2}{|c|}{ Noise intensity (3\%) } \\
\hline & & & & Actual output & Relative error (\%) & Actual output & Relative error (\%) \\
\hline \multirow{4}{*}{1} & \multirow{2}{*}{50} & 20 & 0.5 & 0.457 & 8.57 & 0.48937 & -2.13 \\
\hline & & 30 & 0.5 & 0.534 & -6.74 & 0.52943 & 5.89 \\
\hline & \multirow{2}{*}{70} & 20 & 0.7 & 0.712 & -1.76 & 0.6847 & -2.19 \\
\hline & & 30 & 0.7 & 0.727 & -3.91 & 0.7735 & 10.50 \\
\hline \multirow{4}{*}{4} & \multirow{2}{*}{50} & 20 & 0.5 & 0.492 & 1.50 & 0.47049 & -5.90 \\
\hline & & 30 & 0.5 & 0.468 & 6.39 & 0.45675 & -8.65 \\
\hline & \multirow{2}{*}{70} & 20 & 0.7 & 0.769 & -9.81 & 0.76837 & 9.77 \\
\hline & & 30 & 0.7 & 0.743 & -6.15 & 0.71513 & 2.16 \\
\hline \multirow{4}{*}{5} & \multirow{2}{*}{50} & 20 & 0.5 & 0.491 & 1.74 & 0.41528 & -16.94 \\
\hline & & 30 & 0.5 & 0.528 & -5.53 & 0.41918 & -16.16 \\
\hline & \multirow{2}{*}{70} & 20 & 0.7 & 0.740 & -5.67 & 0.72871 & 4.10 \\
\hline & & 30 & 0.7 & 0.738 & -5.40 & 0.72388 & 3.41 \\
\hline
\end{tabular}

TABLE 10: The identification results of damage locations when two braces are damaged with noises.

\begin{tabular}{|c|c|c|c|c|c|c|c|c|}
\hline \multirow{3}{*}{ Damage scenarios } & \multirow{3}{*}{$a_{j}(\%)$} & \multirow{3}{*}{$T\left({ }^{\circ} \mathrm{C}\right)$} & \multicolumn{6}{|c|}{ Output nodes } \\
\hline & & & \multicolumn{3}{|c|}{ Noise intensity (1\%) } & \multicolumn{3}{|c|}{ Noise intensity (3\%) } \\
\hline & & & First & Second & Third & First & Second & Third \\
\hline \multirow{4}{*}{ DC 4} & \multirow[b]{2}{*}{50} & 20 & $0.999987(1)$ & $1.99 \times 10^{-6}(0)$ & $1.34 \times 10^{-4}(0)$ & $0.999997(1)$ & $0.000372(0)$ & $3.37 \times 10^{-6}(0)$ \\
\hline & & 30 & $0.999999(1)$ & $3.69 \times 10^{-6}(0)$ & $1.96 \times 10^{-6}(0)$ & $0.999999(1)$ & $0.000174(0)$ & $9.04 \times 10^{-8}(0)$ \\
\hline & \multirow[b]{2}{*}{70} & 20 & $0.999994(1)$ & $2.97 \times 10^{-6}(0)$ & $3.85 \times 10^{-5}(0)$ & $0.999960(1)$ & $4.00 \times 10^{-5}(0)$ & $5.17 \times 10^{-3}(0)$ \\
\hline & & 30 & $0.999997(1)$ & $5.64 \times 10^{-6}(0)$ & $1.68 \times 10^{-5}(0)$ & $0.999999(1)$ & $2.96 \times 10^{-6}(0)$ & $2.33 \times 10^{-6}(0)$ \\
\hline \multirow{4}{*}{ DC 5} & \multirow{2}{*}{50} & 20 & $7.19 \times 10^{-4}(0)$ & $0.999967(1)$ & $7.14 \times 10^{-8}(0)$ & $0.031974(0)$ & $0.999966(1)$ & $5.06 \times 10^{-8}(0)$ \\
\hline & & 30 & $2.61 \times 10^{-2}(0)$ & $0.999996(1)$ & $1.16 \times 10^{-8}(0)$ & $0.011941(0)$ & $0.999970(1)$ & $6.86 \times 10^{-8}(0)$ \\
\hline & \multirow{2}{*}{70} & 20 & $2.61 \times 10^{-8}(0)$ & $0.999855(1)$ & $4.84 \times 10^{-4}(0)$ & $5.12 \times 10^{-8}(0)$ & $0.999957(1)$ & $1.68 \times 10^{-3}(0)$ \\
\hline & & 30 & $1.08 \times 10^{-8}(0)$ & $1(1)$ & $4.50 \times 10^{-4}(0)$ & $0.000281(0)$ & 0.999975 (1) & $1.86 \times 10^{-6}(0)$ \\
\hline \multirow{4}{*}{ DC 6} & \multirow{2}{*}{50} & 20 & $2.16 \times 10^{-6}(0)$ & $1.63 \times 10^{-6}(0)$ & $0.999998(1)$ & $0.086943(0)$ & $1.16 \times 10^{-6}(0)$ & 0.998469 (1) \\
\hline & & 30 & $1.92 \times 10^{-6}(0)$ & $1.63 \times 10^{-6}(0)$ & 0.999998 (1) & $0.000482(0)$ & $1.36 \times 10^{-6}(0)$ & $0.999981(1)$ \\
\hline & \multirow{2}{*}{70} & 20 & $2.67 \times 10^{-5}(0)$ & $1.32 \times 10^{-6}(0)$ & $0.999982(1)$ & $0.306331(0)$ & $8.76 \times 10^{-6}(0)$ & $0.990272(1)$ \\
\hline & & 30 & $7.32 \times 10^{-3}(0)$ & $5.53 \times 10^{-6}(0)$ & $0.989758(1)$ & $0.228254(0)$ & $8.42 \times 10^{-6}(0)$ & $0.796207(1)$ \\
\hline
\end{tabular}

The target results of networks are shown in the parentheses.

TABLE 11: The identification results of damage severities when two braces are damaged and contaminated with noises.

\begin{tabular}{|c|c|c|c|c|c|c|c|c|c|c|c|c|}
\hline \multirow[b]{2}{*}{ Damaged braces } & \multirow[b]{2}{*}{$a_{j}(\%)$} & \multirow[b]{2}{*}{$T\left({ }^{\circ} \mathrm{C}\right)$} & \multirow{2}{*}{\multicolumn{2}{|c|}{$\begin{array}{l}\text { Target } \\
\text { output }\end{array}$}} & \multicolumn{4}{|c|}{ Noise intensity (1\%) } & \multicolumn{4}{|c|}{ Noise intensity (3\%) } \\
\hline & & & & & \multicolumn{2}{|c|}{ Actual output } & \multicolumn{2}{|c|}{ Relative error } & \multicolumn{2}{|c|}{ Actual output } & \multicolumn{2}{|c|}{$\begin{array}{c}\text { Relative } \\
\text { error }\end{array}$} \\
\hline \multirow{4}{*}{1,4} & \multirow{2}{*}{50} & 20 & 0.5 & 0.5 & 0.50956 & 0.50752 & 1.91 & 1.50 & 0.52651 & 0.52599 & 5.30 & 5.20 \\
\hline & & 30 & 0.5 & 0.5 & 0.5244 & 0.52215 & 4.88 & 4.43 & 0.54715 & 0.54677 & 9.43 & 9.35 \\
\hline & \multirow[b]{2}{*}{70} & 20 & 0.7 & 0.7 & 0.71206 & 0.71533 & 1.72 & 2.19 & 0.76501 & 0.76407 & 9.29 & 9.15 \\
\hline & & 30 & 0.7 & 0.7 & 0.74194 & 0.74332 & 5.99 & 6.19 & 0.79502 & 0.79349 & 13.57 & 13.36 \\
\hline \multirow{4}{*}{1,5} & \multirow{2}{*}{50} & 20 & 0.5 & 0.5 & 0.41313 & 0.41148 & 17.37 & 17.70 & 0.47940 & 0.47879 & 4.12 & 4.24 \\
\hline & & 30 & 0.5 & 0.5 & 0.45802 & 0.45692 & 8.40 & 8.62 & 0.49173 & 0.49111 & 1.65 & 1.78 \\
\hline & \multirow{2}{*}{70} & 20 & 0.7 & 0.7 & 0.61038 & 0.61457 & 12.80 & 12.20 & 0.5861 & 0.58614 & 16.27 & 16.26 \\
\hline & & 30 & 0.7 & 0.7 & 0.73215 & 0.73307 & 4.59 & 4.72 & 0.62806 & 0.62811 & 10.28 & 10.27 \\
\hline \multirow{4}{*}{4,5} & \multirow{2}{*}{50} & 20 & 0.5 & 0.5 & 0.39151 & 0.3929 & 21.70 & 21.42 & 0.45318 & 0.45306 & 9.36 & 9.39 \\
\hline & & 30 & 0.5 & 0.5 & 0.45248 & 0.4539 & 9.50 & 9.22 & 0.43403 & 0.43389 & 13.19 & 13.22 \\
\hline & \multirow{2}{*}{70} & 20 & 0.7 & 0.7 & 0.6481 & 0.64835 & 7.41 & 7.38 & 0.72292 & 0.72244 & 3.28 & 3.21 \\
\hline & & 30 & 0.7 & 0.7 & 0.68631 & 0.68668 & 1.96 & 1.90 & 0.72519 & 0.72474 & 3.60 & 3.53 \\
\hline
\end{tabular}

from Table 11, the maximum values of relative error for $1 \%$ and $3 \%$ noise levels are $21.70 \%$ and $16.27 \%$, respectively. However, the average values of relative errors for all cases are less than $8.5 \%$, and the majority of relative errors is less than $10 \%$, which implies that the proposed approach can identify the multiple damage severities with reasonable accuracy for the noise with high level. As a consequence, the proposed approach can still produce a robust diagnosis regardless of $3 \%$ noise pollution level, identifying damage locations and severities with reasonable accuracy even when temperature variations and noise contamination are both considered. 


\section{Conclusion}

In this paper, a novel two-step damage identification strategy is proposed to compensate adverse temperature effects by using only acceleration responses and temperature data. Differences in the first three-order coefficients of the AR time series modeling vibration data are extracted as the damage features. In the first step, BPNN models, which output the damage locations or patterns after feeding those damage features and temperatures, are formulated to localize damage. In the second step, another BPNN model, fed into the same inputs, is established to identify damage severities. It is proven that, for the steel-braced frame FE model, this approach can successfully identify the locations and severities in single and multiple damage cases with reasonable accuracy under varying temperature conditions. Temperature effects are effectively compensated for all damage scenarios even when the noise intensity is up to $3 \%$. Furthermore, this method not only requires small sample data but also fulfills more reliable damage identifications without false diagnoses. Therefore, the propose method using output-only vibration data has great potential for practical applications, as it is so robust, reliable, and precise in identifying damage in the presence of temperature variations and noise interference. In reality, changing environmental and operation conditions such as loading, boundary conditions, and moisture also inevitably affects measured signals of structures, resulting in inability of the proposed method. Additionally, the training dataset should be collected over a wide range of temperature conditions to eschew false diagnosis. Further research based on experimental tests from real structures with multitype structural damage cases should be conducted to make the introduced identification approach more applicable for practical engineering.

\section{Data Availability}

The data used to support the findings of this study are available from the corresponding author upon request.

\section{Conflicts of Interest}

The authors declare that there are no conflicts of interest regarding the publication of this paper.

\section{Acknowledgments}

This study was supported by the Science and Technology Research Planning Project (2018), Educational Commission of Hubei Province, China (no. B2018051).

\section{References}

[1] S. Das, P. Saha, and S. K. Patro, "Vibration-based damage detection techniques used for health monitoring of structures: a review," Journal of Civil Structural Health Monitoring, vol. 6, no. 3, pp. 477-507, 2016.

[2] L. Mao, S. Weng, S.-J. Li, H.-P. Zhu, and Y.-H. Sun, "Statistical damage identification method based on dynamic response sensitivity," Journal of Low Frequency Noise, Vibration and Active Control, Article ID 1461348418784828, 2018.

[3] S. V. Varpe and D. Ainchwar, "A survey on vibration based damage detection of bridges under varying temperature effects using time series analysis," International Journal of Innovative Research in Science, Engineering and Technology, vol. 7, no. 1, pp. 1065-1073, 2018.

[4] C. Liu and J. T. DeWolf, "Effect of temperature on modal variability of a curved concrete bridge under ambient loads," Journal of Structural Engineering, vol. 133, no. 12, pp. 17421751, 2007.

[5] H. F. Zhou, Y. Q. Ni, J. M. Ko, and K. Y. Wong, "Modeling of wind and temperature effects on modal frequencies and analysis of relative strength of effect," Wind and Structures, vol. 11, no. 1, pp. 35-50, 2008.

[6] I. Dominik, M. Iwaniec, and $Ł$. Lech, "Low frequency damage analysis of electric pylon model by fuzzy logic application," Journal of Low Frequency Noise, Vibration and Active Control, vol. 32, no. 3, pp. 239-251, 2013.

[7] J. Xu, J. Hao, H. Li et al., "Experimental damage identification of a model reticulated shell," Applied Sciences, vol. 7, no. 4, 362 pages, 2017.

[8] A. A. Jiménez, C. Q. G. Muñoz, and F. P. G. Márquez, "Machine learning for wind turbine blades maintenance management," Energies, vol. 11, no. 1, 13 pages, 2017.

[9] F. Z. Tajrishi, A. M. G. Roshan, M. Zeynalian, and J. V. Amiri, "A new collection of compressed damage indices for multidamage detection of cold formed steel shear walls based on neural network ensembles," Canadian Journal of Civil Engineering, vol. 43, no. 12, pp. 1034-1043, 2016.

[10] J. Min, S. Park, C.-B. Yun, C.-G. Lee, and C. Lee, "Impedancebased structural health monitoring incorporating neural network technique for identification of damage type and severity," Engineering Structures, vol. 39, pp. 210-220, 2012.

[11] F. Shabbir, M. Khan, N. Ahmad et al., "Structural damage detection with different objective functions in noisy conditions using an evolutionary algorithm," Applied Sciences, vol. 7, no. 12, 1245 pages, 2017.

[12] J. Vitola, F. Pozo, D. Tibaduiza, and M. Anaya, "Distributed piezoelectric sensor system for damage identification in structures subjected to temperature changes," Sensors, vol. 17, no. 6, 1252 pages, 2017.

[13] Y. Xia, B. Chen, S. Weng, Y.-Q. Ni, and Y.-L. Xu, "Temperature effect on vibration properties of civil structures: a literature review and case studies," Journal of Civil Structural Health Monitoring, vol. 2, no. 1, pp. 29-46, 2012.

[14] Y. Xia, H. Hao, G. Zanardo, and A. Deeks, "Long term vibration monitoring of an RC slab: temperature and humidity effect," Engineering Structures, vol. 28, no. 3, pp. 441-452, 2006.

[15] H. Sohn, M. Dzwonczyk, E. G. Straser et al., “An experimental study of temperature effect on modal parameters of the Alamosa Canyon Bridge," Earthquake Engineering and Structural Dynamics, vol. 28, no. 8, pp. 879-897, 1999.

[16] J. P. Amezquita-Sanchez and H. Adeli, "Synchrosqueezed wavelet transform-fractality model for locating, detecting, and quantifying damage in smart highrise building structures," Smart Materials and Structures, vol. 24, no. 6, Article ID 065034, 2015.

[17] Y. Ying, J. H. Garrett Jr, I. J. Oppenheim et al., "Toward datadriven structural health monitoring: application of machine learning and signal processing to damage detection," Journal of Computing in Civil Engineering, vol. 27, no. 6, pp. 667-680, 2013. 
[18] M.-S. Huang, M. Gül, and H.-P. Zhu, "Vibration-based structural damage identification under varying temperature effects," Journal of Aerospace Engineering, vol. 31, no. 3, Article ID 04018014, 2018.

[19] Z. Chen and L. Yu, "A novel PSO-based algorithm for structural damage detection using Bayesian multi-sample objective function," Structural Engineering and Mechanics, vol. 63 , no. 6, pp. 825-835, 2017.

[20] M. S. Huang, S. X. Cheng, H. L. Lu, M. Gul, and H. Zhang, "Structural damage identification of steel-concrete composite bridge under temperature effects based on Cuckoo Search," International Journal of Lifecycle Performance Engineering, vol. 3, no. 2, pp. 111-130, 2019.

[21] J. F. Gu, M. Gul, and X. Wu, "Damage detection under varying temperature using artificial neural networks," Structural Control and Health Monitoring, vol. 24, no. 11, e1998 pages, 2017.

[22] B. Kostić and M. Gül, "Vibration-based damage detection of bridges under varying temperature effects using time-series analysis and artificial neural networks," Journal of Bridge Engineering, vol. 22, no. 10, Article ID 04017065, 2017.

[23] H. T. Chen, S. X. Jiang, X. H. Wang, and Z. Y. Wang, "Fault diagnosis of wind turbine gearbox based on wavelet neural network," Journal of Low Frequency Noise, Vibration and Active Control, vol. 37, no. 4, pp. 977-986, 2018.

[24] H. F. Zhou, Y. Q. Ni, and J. M. Ko, "Structural damage alarming using auto-associative neural network technique: exploration of environment-tolerant capacity and setup of alarming threshold," Mechanical Systems and Signal Processing, vol. 25, no. 5, pp. 1508-1526, 2011.

[25] R. P. Bandara, Damage identification and condition assessment of building structures using frequency response functions and neural networks, Ph.D. thesis, Queensland University of Technology, Brisbane, Australia, 2013.

[26] H. Sohn, K. Worden, and C. R. Farrar, "Statistical damage classification under changing environmental and operational conditions," Journal of Intelligent Material Systems and Structures, vol. 13, no. 9, pp. 561-574, 2002.

[27] M. Rezaiee-Pajand, A. Entezami, and H. Shariatmadar, "An iterative order determination method for time-series modeling in structural health monitoring," Advances in Structural Engineering, vol. 21, no. 2, pp. 300-314, 2018.

[28] G. Prakash and S. Narasimhan, "Bayesian two-phase gamma process model for damage detection and prognosis," Journal of Engineering Mechanics, vol. 144, no. 2, Article ID 04017158, 2018.

[29] L. Bornn, C. R. Farrar, G. Park, and K. Farinholt, "Structural health monitoring with autoregressive support vector machines," Journal of Vibration and Acoustics, vol. 131, no. 2, Article ID 021004, 2009.

[30] M. Mollineaux and R. Rajagopal, "Structural health monitoring of progressive damage," Earthquake Engineering and Structural Dynamics, vol. 44, no. 4, pp. 583-600, 2015.

[31] A. J. Wootton, J. B. Butcher, T. Kyriacou, C. R. Day, and P. W. Haycock, "Structural health monitoring of a footbridge using Echo State Networks and NARMAX," Engineering Applications of Artificial Intelligence, vol. 64, pp. 152-163, 2017.

[32] P. C. Young, Recursive Estimation and Time-Series Analysis: An Introduction, Springer Science \& Business Media, B.V. Press, Dordrecht, Netherlands, 2012.

[33] C. Francq and A. Gautier, "Large sample properties of parameter least squares estimates for time-varying arma models," Journal of Time Series Analysis, vol. 25, no. 5, pp. 765-783, 2004.

[34] G. Eshel, "The yule walker equations for the AR coefficients," Internet Resource, vol. 2, pp. 68-73, 2003.

[35] Y. Wang, S. Kang, Y. Jiang et al., "Classification of fault location and the degree of performance degradation of a rolling bearing based on an improved hyper-sphere-structured multi-class support vector machine," Mechanical Systems and Signal Processing, vol. 29, pp. 404-414, 2012.

[36] K. K. Nair, A. S. Kiremidjian, and K. H. Law, "Time seriesbased damage detection and localization algorithm with application to the ASCE benchmark structure," Journal of Sound and Vibration, vol. 291, no. 1-2, pp. 349-368, 2006.

[37] S. J. Dyke, D. Bernal, J. L. Beck, and C. Ventura, “An experimental benchmark problem in structural health monitoring," in Proceedings of the 3rd International Workshop on Structural Health Monitoring, pp. 488-497, Stanford, CA, USA, 2001.

[38] A.-M. Yan, G. Kerschen, P. D. Boe, and J.-C. Golinval, "Structural damage diagnosis under varying environmental conditions-part II: local PCA for non-linear cases," Mechanical Systems and Signal Processing, vol. 19, no. 4, pp. 847-864, 2005. 